\title{
High Temperature Condensed Phase Mass Spectrometric Analysis Program \\ Final Report
}

\section{Summary}

This program (EMSP Project \#60424) was funded by the EM Science Program for the development of an integrated mass spectrometric analysis system (see Figure 1.) capable of analyzing materials from room up to high temperatures, with the practical upper temperature limit to be experimentally determined. A primary objective of the program was the development of techniques to analyze waste materials during vitrification processing to produce waste forms.

The sample is heated in the ion formation region of the mass spectrometer. This instrument geometry allows the atoms and molecules that volatilize from the sample as neutrals to be ionized before they have a chance to condense on surfaces that generally are cooler that the sample. In addition, this geometry facilitates more efficient focussing of SIMS and thermal ions into the quadrupole mass analyzer. Instrumental capabilities include the detection of volatilizing neutral species by electron bombardment, ions forming on the surface by surface ionization, and surface species by static SIMS. In addition, the instrument has elemental analysis capability (by dynamic SIMS).

It was also initially proposed that during the concluding year the instrument would be used for a series of demonstration analyses; however, the program was funded at just $69 \%$ of the requested level, which proved adequate to complete the major aspects of the instrument development but was insufficient for the complete development of the dynamic SIMS capability and the completion of the demonstration analyses. The demonstration analyses are being performed with the support of other programs in our laboratory, and a dynamic SIMS gun, which was made available from another of our 
programs, is installed on the instrument (but has not yet been tested). Demonstration analyses have proven the concept of the instrument; all of the capabilities, other than the dynamic SIMS capability, have been successfully deployed. The dynamic SIMS capability will be more fully developed when another program has a demonstrated need and funds that activity.

The practical upper temperature limit for analyses is proving to be limited by mechanical stability of the sample support hardware at high temperatures and by chemical reactions between the material to be analyzed and the supporting filament or cup. The practical upper temperature limit for mechanical stability for the filament mode is proving to be about $1700^{\circ} \mathrm{C}$, while for the refractory metal cup mode the limit is about $1300^{\circ} \mathrm{C}$. When chemical reactions occur between the material and the cup or filament, which is often the case, the temperature limit can be lower, although systems have been studied at above $1000^{\circ} \mathrm{C}$. In general, the cup assembly is proving to be more resistant to failure due to chemical reactions since the sample is not in contact with the delicate filament (that can be eroded through chemical reactions with the sample). The ability to study chemical interactions between the sample and the holder is turning out to be a significant advantage offered by the instrument, since this allows potential corrosion reactions in glass melters to be identified and analyzed.

The specially designed ion source combines all of the ionization modes into a unified design so that a single sample can be analyzed in all of these modes in rapid sequence as the temperature is ramped upward, with a special emphasis on the ionization of neutral vapors prior to condensation. This permits the analysis of high temperature materials prior to condensing on the much cooler walls of the source housing.

The computer controlled data system allows rapid sequencing between the ionization modes so that various processes can be 
monitored as the temperature is ramped upward. The types of information that are given to the computer are: the desired temperature ramp, the ionization modes and the parameters for controlling those modes, and the masses to be analyzed within each of the ionization modes. The computer increases the current going to the sample heater in the pre-programmed fashion, reads the temperature via a thermocouple, sequences between the specified masses for the chosen ionization modes and stores the time, temperature, current supplied to the heater, mass, and ion intensities for the chosen masses for each ionization mode that is specified.

The data files are constructed to facilitate easy downloading to spreadsheet or plotting applications at the completion of data collection. A typical analysis could result in a plot of the various gases emanating from a sample plotted as a function of temperature, followed by plotting a particular off-gas event overlaying the appearance or disappearance of a particular ion during SIMS analysis. In this way various ionization modes can be used to gain insights into chemical reactions that occur in materials during thermal cycles.

While it is not possible to collect the ion intensities in all ionization modes simultaneously, the interleaved data collection process makes it possible to collect the data rapidly enough for the data points in the plots to be sufficiently close to detect phase changes. This is an excellent method for detecting phenomena corresponding to changes in the sample with temperature, and can be used to complement data from differential thermal analyses and other related analytical techniques.

The instrument is currently being used to determine the chemical changes that take place during the melting of the components of various glasses, which includes the evolution of gases and vapors as well as the change in molecular species in the melt. In these experiments it will be possible to study the correlation of the emission of ions and neutrals from the glass by detecting the species that effuse from the sample (neutral species by electron impact 
ionization followed by ion detection and thermal ions by direct detection).

\section{Introduction}

The conceptual basis for this program came from our continuing efforts to better characterize and understand the physical and chemical processes that govern the emission of ions from high temperature materials. In particular it relates to our attempts to better characterize the chemical and physical processes that govern the emission of thermal ions from molten glass ion emitters, which is the method of choice for the isotope ratio analysis of about 30 elements.[1-4] We had developed instrumentation to allow the analysis of ions, permanent gases and condensable vapors from a variety of glasses; however, this capability was spread between two different instruments and the data collection was too slow to allow accurate monitoring of the temperature verses ion intensity curves for more than one ionization mode at a time. $[1,5,6]$ Thus, in order for a complete analysis to be obtained, two or more samples of the same material had to be analyzed, significantly complicating the analysis as well as introducing uncertainty into the analyses (due to the fact that the same sample could not be analyzed by each method). The temperature ramp on each instrument was manually controlled, further exacerbating the comparison of data. It was also realized that an instrument that combined the different ionization modes into a single instrument, and in which the modes could be rapidly sequenced, could greatly enhance the accuracy with which chemical changes that occurred during the heating process could be correlated. Another need was an even and reproducible heating rate of the sample, which was a significant problem with the older instruments.

It was realized that the same capabilities that were needed for the analysis of these ion emitting materials were essentially the same capabilities needed for the analysis of any material being melted into a glass or other form, including glass and ceramic waste forms for 
waste management activities. This is the background for the proposal that led to the funding of this program.

\section{Instrument Design, Construction and Operation}

The major components of the instrument (see Figure 2.) are the vacuum housing, pumps, ion guns, ion source optics, and mass analyzer.

A year long effort went into the design and construction of the ion source optics (see Figure 3.) that combines all of the stated ion formation modes into a single compact unit. Many designs were modeled with the latest version of the ion optic modeling code "SIMION,"[7] and one design proved to be superior. After extensive "fine tuning" efforts, the ion source design was integrated into an instrument design that was both affordable and flexible. The final design was challenging to build, and required the efforts of a highly skilled craftsman, but it was built and functions exceptionally well. It is fully bakeable, allowing it to be used in an ultrahigh vacuum environment. The sensitivity in all modes is quite high, with the electron bombardment ionization (EI) detection sensitivity (for volatilizing neutral species) being exceptional. This part of the ion source is quite unique, and represents a design breakthrough for EI sources. It is also unique in the way in which the various ion formation modes are integrated into the design.

Much thought went into the type of mass spectrometer to use for the analyzer. There were many trade-offs between the different designs, with quadrupole, ion trap, time of flight and sector instruments considered. A high end quadrupole mass spectrometer was chosen as the most logical choice. The reasons for this choice were the ease with which this type of ion source could be interfaced to the quadrupole analyzer and the fact that we had previously developed a highly versatile computer controlled data system that could be readily modified to accommodate new requirements. It is far more difficult to interface this type of ion source to the other mass spectrometer 
analyzers, and the data systems could not have been customized to the extent that we were able to accomplish with the quadrupole based analyzer. It was desirable to have the sample held in place by gravity, since many materials that are melted into glasses start as powders. This constrained the sample to be held upright, and the instrument to be mounted vertically. The quadrupole design could accommodate any configuration. The ion trap could also be mounted in any configuration, but the data acquisition and instrument control software available for the ion trap based systems could not be configured to perform the planned experiments. Hence the conclusion that the quadrupole was the most feasible mass spectrometer for this application.

The ion source optics has penetrations along 5 axes going from one side to the other, one axis in line with the quadrupole and the vacuum lock, and the other four each at $35^{\circ}$ (off vertical) to the first one and $90^{\circ}$ to each other. (see Figure3.) Two of these are for the two SIMS guns to reach the sample, one for a light that shines on the sample when it is in position, and the other for viewing the sample in position. The face of the sample is located at the intersection of these 5 axes, which is the primary focal point of the lens and the center of the spherical vacuum housing. Opposing ports on the vacuum housing line up with the four axes that are $35^{\circ}$ off vertical.

Predecessor instruments constructed in our laboratory had serious limitations with sample handling due to samples being mounted horizontally, allowing samples to fall from the mount. For this reason, the instrument was mounted in a vertical position so that samples would be held in place by gravity. This complicated the instrument design, but allowed much improved versatility in sample types and mounting.

The sample mounting and heating system has been evolving in our lab for many years, and is well proven. Two versions are in use, one using a filament and the other a tube. The filament version uses a 
miniaturized version of a single filament thermal ionization source, with the sample mounted on the face of the filament. The face of the filament is placed at the main focal point of the ion source. The tube version[6] has a tube mounted on rhenium filaments. The sample is packed into the tube prior to being spot welded onto the supporting filaments. Both types of sample mounting systems are heated by passing current through the filament; in the case of the filament mount the current passes through the single filament supporting the sample, while in the case of the tube the current passes through one filament to the tube and then on to the opposing filament. The tube is more difficult to build and does not have the high temperature mechanical stability of the filament, but allows substantial quantities of material to be packed in. The filament requires samples that adhere to the surface without flaking off, while the tube can accommodate materials that are of a powdery nature. Materials that are going to be melted into a glass can generally be adhered to a filament by gently heating in air until it fuses to the filament, taking care not to heat to the point where the material undergoes reaction; this heating can be performed under an inert gas cover if necessary. Tubes can also be used for melting glasses, and in this case stainless steel tubes work as well as refractory metal tubes since the glasses do not seem to corrode the stainless steel at temperatures up to slightly higher than $1000^{\circ} \mathrm{C}$; for higher temperatures more refractory tubes (such as Re, Pt or Ta) can be employed. A thermocouple is spot welded to the side of the tube or the back of the filament.

The tube or filament source is mounted on a one inch diameter vacuum lock probe that has two electrical power feedthroughs and two thermocouple feedthroughs. The probe is inserted through the vacuum lock mounted on the bottom of the vacuum housing and the sample is inserted up to the focal point of the ion source, using visual observation. The vacuum lock is a variation of the units used for thermal ionization mass spectrometers. It takes about five minutes to insert a sample into the instrument, but it takes much longer than that to get the assembly completed and ready to insert. A steady hand and 
patience are required to assemble a sample mount with the thermocouple properly attached, but it can be accomplished with practice.

The static SIMS gun is a design used in our lab for a number of years and uses the perrhenate anion as the primary projectile.[5] [8] This primary ion is well established as an excellent probe molecule for static SIMS analysis of electrically insulating surfaces, in particular for the analysis of molecular species on the surface.

All of these components are mounted in a spherical vacuum housing (see Figure 2.) designed to be compatible with ultra high vacuum. The probe comes up through the bottom, and the quadrupole mass spectrometer is opposite the source (on the top side). The face of the sample sits in the ion source housing at the focal point of the lens, which also happens to be the center of the spherical vacuum housing. The ion source housing has four line of sight penetrations going from one side and out the other, one for the static SIMS gun, one for the dynamic SIMS gun, one for a light and the fourth for viewing. All of these penetrations are at a $35^{\circ}$ angle to the axis of the sample and the mass spectrometer. Opposite the static SIMS gun is a retractable Faraday cup and a microchannel plate image intensifier. The dynamic SIMS gun is mounted on an equivalent port. A light mounted outside the vacuum housing is focused through an access hole into the source, and the fourth access port is used for viewing the sample, either with the naked eye or through a telescope/pyrometer. A telescope style pyrometer is semi-permanently mounted on a viewing port and focused on the plane that includes the mounted sample. In addition to using the instrument for temperature measurement, the pyrometer may also be used to visually inspect the sample during heating, allowing the user to visually determine when melting of the sample has occurred.

When the static SIMS capability is to be used, the primary beam is accelerated through the source and focused using the image 
intensifier. During this time the sample is slightly retracted so that it does not intercept the beam. Then the Faraday cup is inserted and the beam intensity adjusted. Then the sample is inserted so the face is at the focal point of the lens, and the beam is positioned to silhouette the sample on the image intensifier. The beam is then deflected from the sample until the appropriate time for analysis.

The data system, which is a modified version of our "standard" laboratory data control/data acquisition system, is based on and utilizes hardware and software developed in our laboratory over the past ten years. We have full access to the source code (since it was developed by us) and it is a highly modular system readily adaptable to new additions. It controls the power supply that heats the sample, and is interfaced to the thermocouple controller so that the temperature can be read by the computer. The computer has full control over the power supplies that control the focusing voltages and thus can switch focusing conditions for various ionization modes. The computer also has full control over the functions of the mass spectrometer. The software generates the parameters to generate the appropriate temperature ramp. The desired ionization modes are specified, and the masses to be monitored for each ionization mode are specified. The computer controls the parameters for scanning and for switching between ionization modes. The scanning of the specified masses for each specified ionization mode and the temperature ramp are initiated. The computer stores peak heights for each mode along with the time and temperature. At the end of the analysis the data can be down loaded into a spread sheet.

When the data is in the spread sheet it can be manipulated according to the needs of the analysis. Only one peak can be measured for one ionization mode at a time, but sequencing with computer control is fast in comparison to the rate of change in the sample, so the data are collected rapidly enough to track the intensity changes as functions of time and temperature. The spread sheet can then be used to plot ion intensity as a function of temperature for each ion. Those ions that originate from the same parent ion can be summed, if that is 
consistent with the purpose of the analysis and if the temperature profiles are a good overlap. A more usual analysis would involve the verification that the peaks did overlap, and then dropping all but either the most intense one, or the parent ion, in order to clarify the plot. For example, the $\mathrm{NO}_{2}, \mathrm{NO}$ and $\mathrm{O}$ peaks at 46, 30 and $16 \mathrm{amu}$ have identical temperature profiles in the temperature region where nitrate decomposes, so generally only the parent peak at 46 is plotted to simplify the plot. Ion profiles from different modes can also be compared to seek clues to changes in the material of interest with increasing temperature. When the analysis is finished, it can be compared to the results from a DTA or another similar type of thermal analysis.

From the limited number of analyses that have been conducted to date, we believe the most valuable information accessible by our new instrumental approach, that cannot be gained by other means, is the evolution of condensable metal and highly reactive high temperature species from condensed-phase materials. For example, when silver in the elemental form volatilizes from borosilicate glass at temperatures between 900 to $1000^{\circ} \mathrm{C}$ it does so as a mixture of thermal ions and neutral species.[3] Precise temperature profiles were obtained for this process. Silver was subsequently added to the glass as the nitrate, but we were able to show that the silver nitrate decomposed to evolve nitrate over a range from 200 to $400^{\circ} \mathrm{C}$, leaving silver in the elemental state that evolved at the higher temperature. (The thermodynamics of many silver salts are such that it appears that it is not possible for silver to exist in other that this elemental state at high temperatures). The nitrogen oxides are sufficiently reactive to necessitate in situ measurement. Our new instrumental techniques allowed the nitrogen oxides and the silver to be measured on the same sample without having to perform the analyses in two different instruments using successive samples, which would have resulted in the loss of valuable information. A plot of data for silver nitrate in a borosilicate glass collected with the instrument is shown in Figure 4. 


\section{Conclusions}

This successful completion of this program has allowed us to lay the groundwork for the development of an improved technique for analyzing the high temperature chemistry of condensed phase materials. This technique allows the convenient detection of gases and condensable vapors as they evolve from a material undergoing thermal processing, providing temperature profiles of the off gases, while at the same time allowing the user to detect changes in surface chemistry using the static SIMS capability.

This instrument is currently being used to gain new insights into molten glass ion emitters for a BES program, to understand the mechanisms by which ferric nitrate undergoes thermal decomposition for a geochemistry program, and to gain a fundamental understanding of how a thermal ionization method for iodine isotope ratio analyses functions for an environmental program. There are many small, individual analyses that we have concocted that we plan to conduct with this instrument in the next year and beyond, benefiting a variety of programs. We have also opened dialogs with other researchers at other labs that are developing glass and ceramic processes for the storage of waste, but as of this writing none have reached the stage where actual laboratory work is being conducted. We are negotiating to enter into joint programs with other labs for this type of study in the coming year.

\section{References}

1 J. E. Delmore, T. Huett, J. E. Olson, and A. D. Appelhans, International Journal of Mass Spectrometry and Ion Processes, 155 (1996) 149.

2 T. Huett, J. C. Ingram, and J. E. Delmore, International Journal of Mass Spectrometry and Ion Processes, 146/147 (1995) 5.

3 G. F. Kessinger, T. Huett, and J. E. Delmore, submitted to International Journal of Mass Spectrometry, October, 2000.

4 G. F. Kessinger and J. E. Delmore, in preparation for submittal to International Journal of Mass Spectrometry (2001). 
5 J. E. Delmore, A. D. Appelhans, and E. S. Peterson, International Journal of Mass Spectrometry and Ion Processes, 146/147 (1995) 15.

6 J. E. Delmore, A. D. Appelhans, and E. S. Peterson, International Journal of Mass Spectrometry and Ion Processes, 108 (1991) 179.

7 D. A. Dahl, 'SIMION 3D, Version 6.0 User's Manual', INEL95/0403, Idaho National Engineering Laboratory, 1995.

8 D. A. Dahl, A. D. Appelhans, and M. B. Ward, International Journal of Mass Spectrometry, 189 (1999) 39. 


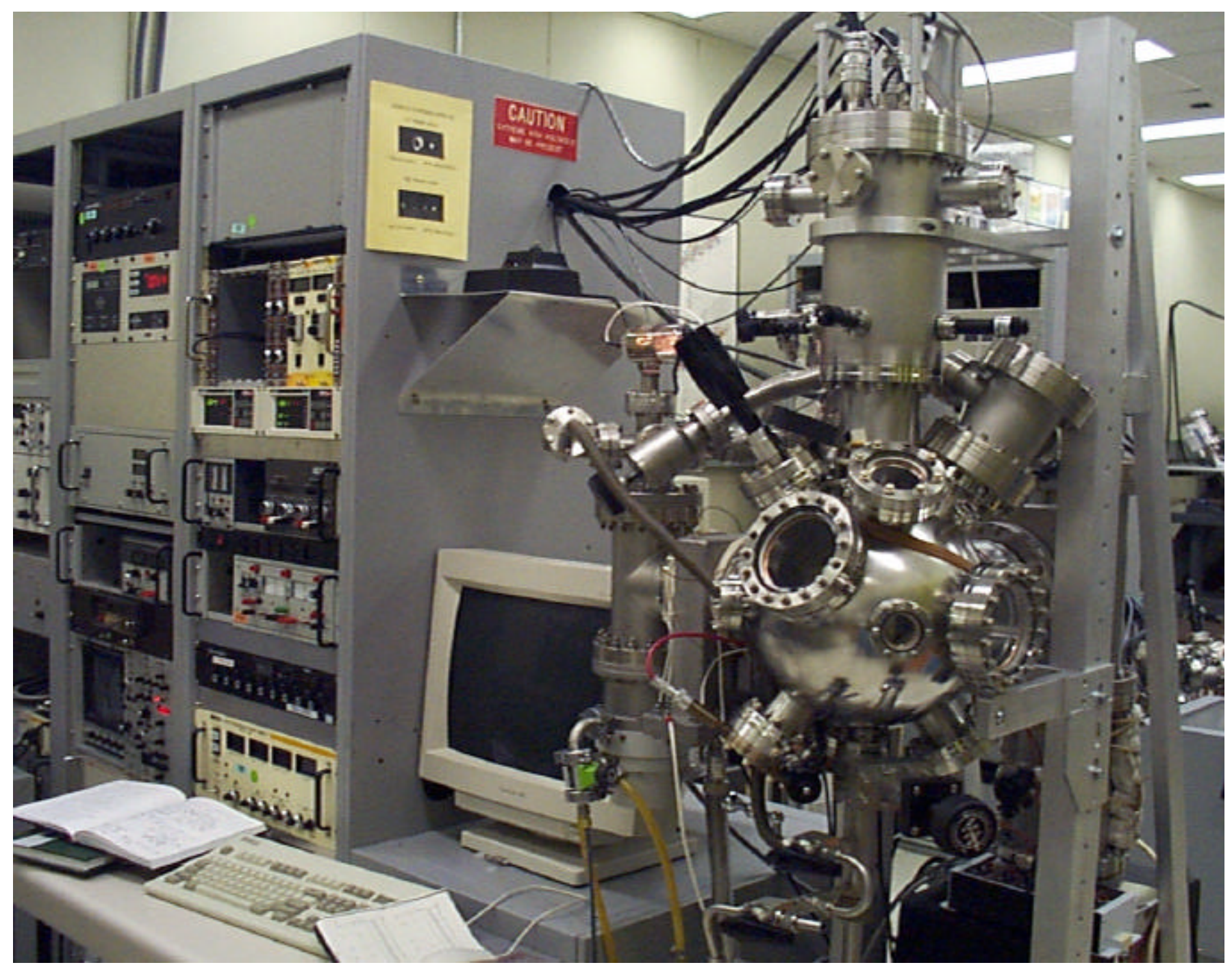

Figure 1. HT-SIMS instrument and peripheral electronic components. 


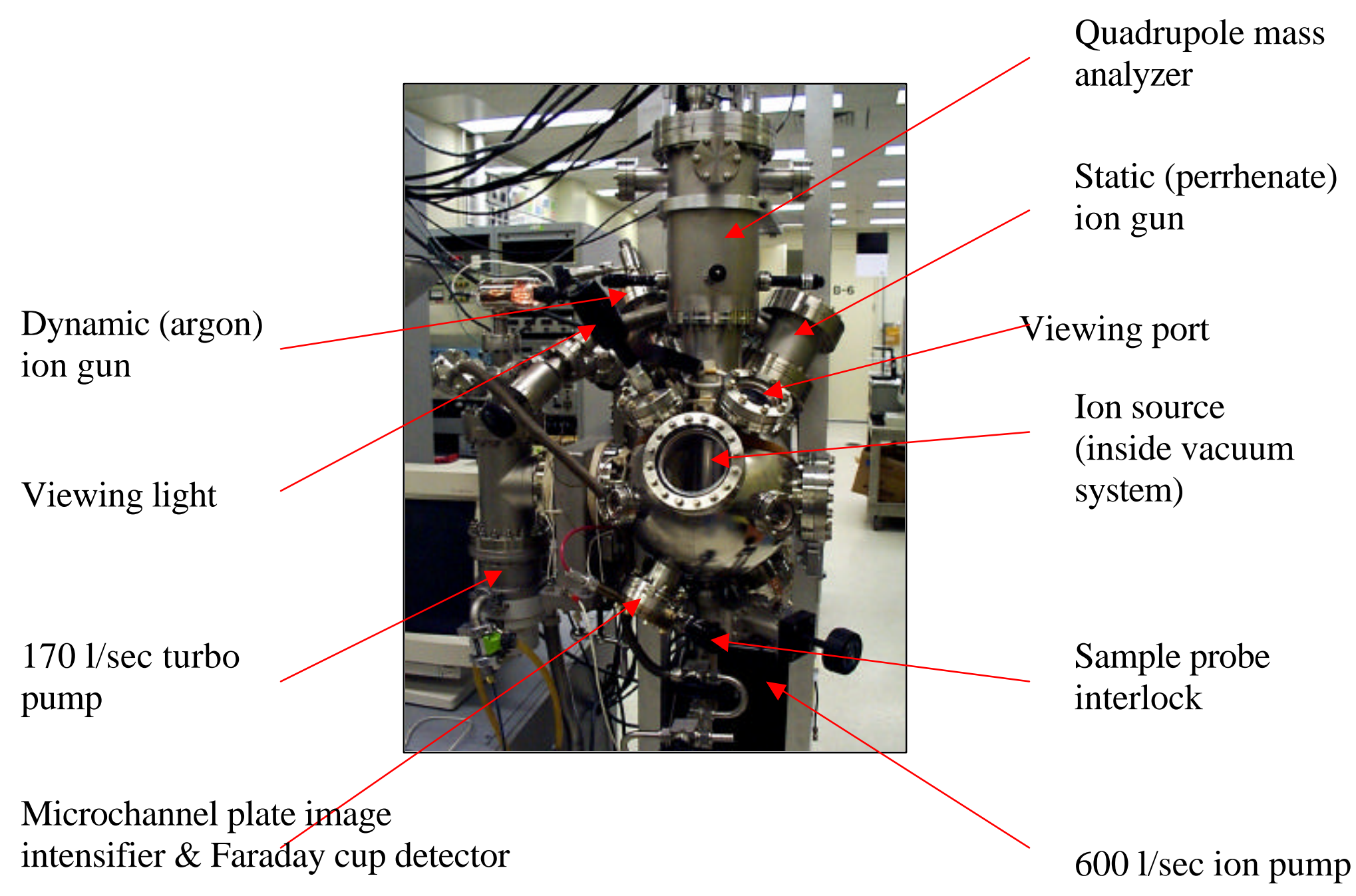

Figure 2. Major components of the HT-SIMS instrument. 


\section{E.I. Ions \\ SIMS Ions}

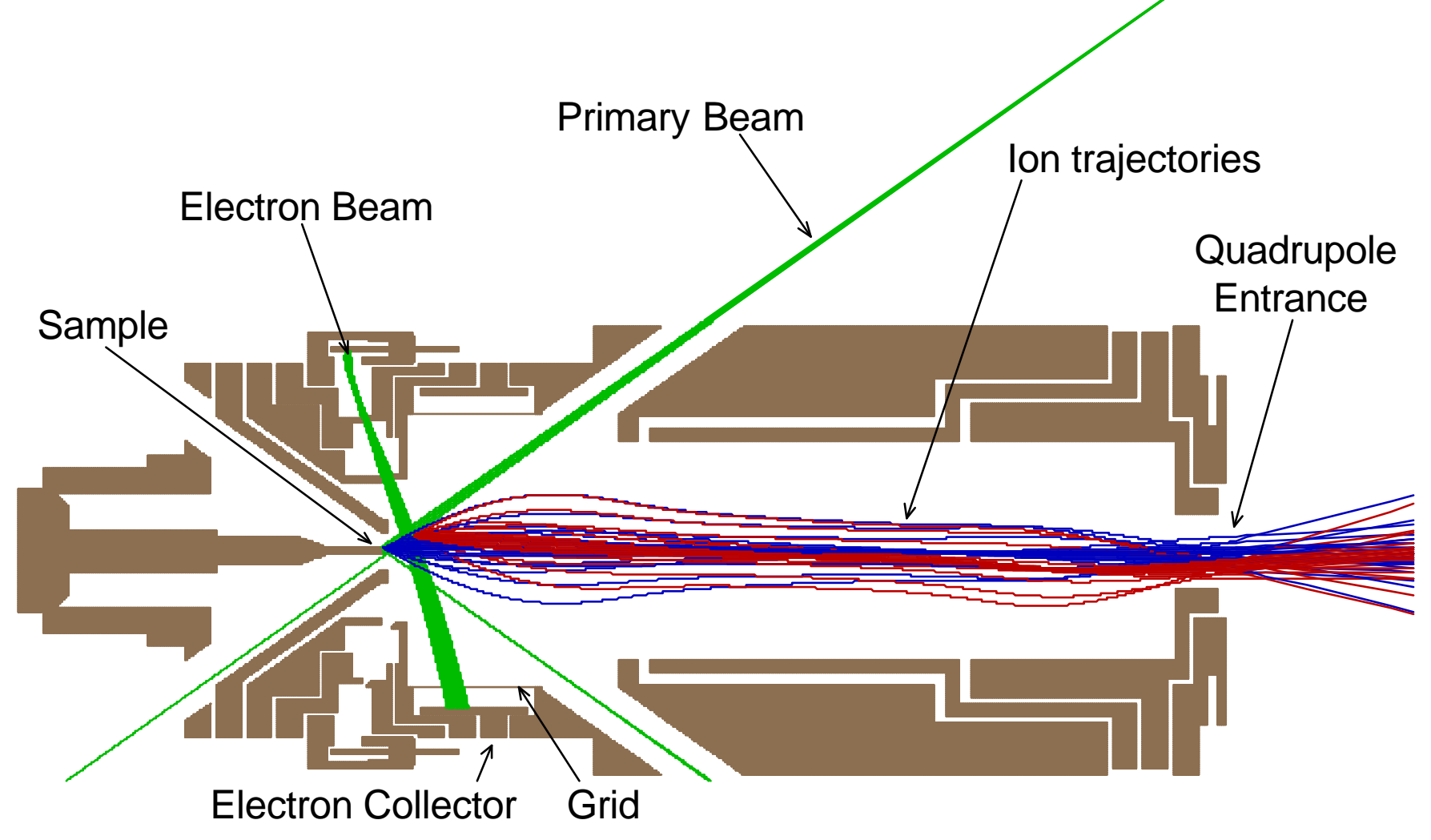

Figure 3. SIMION-generated ion optic cross-section showing ion trajectories. 


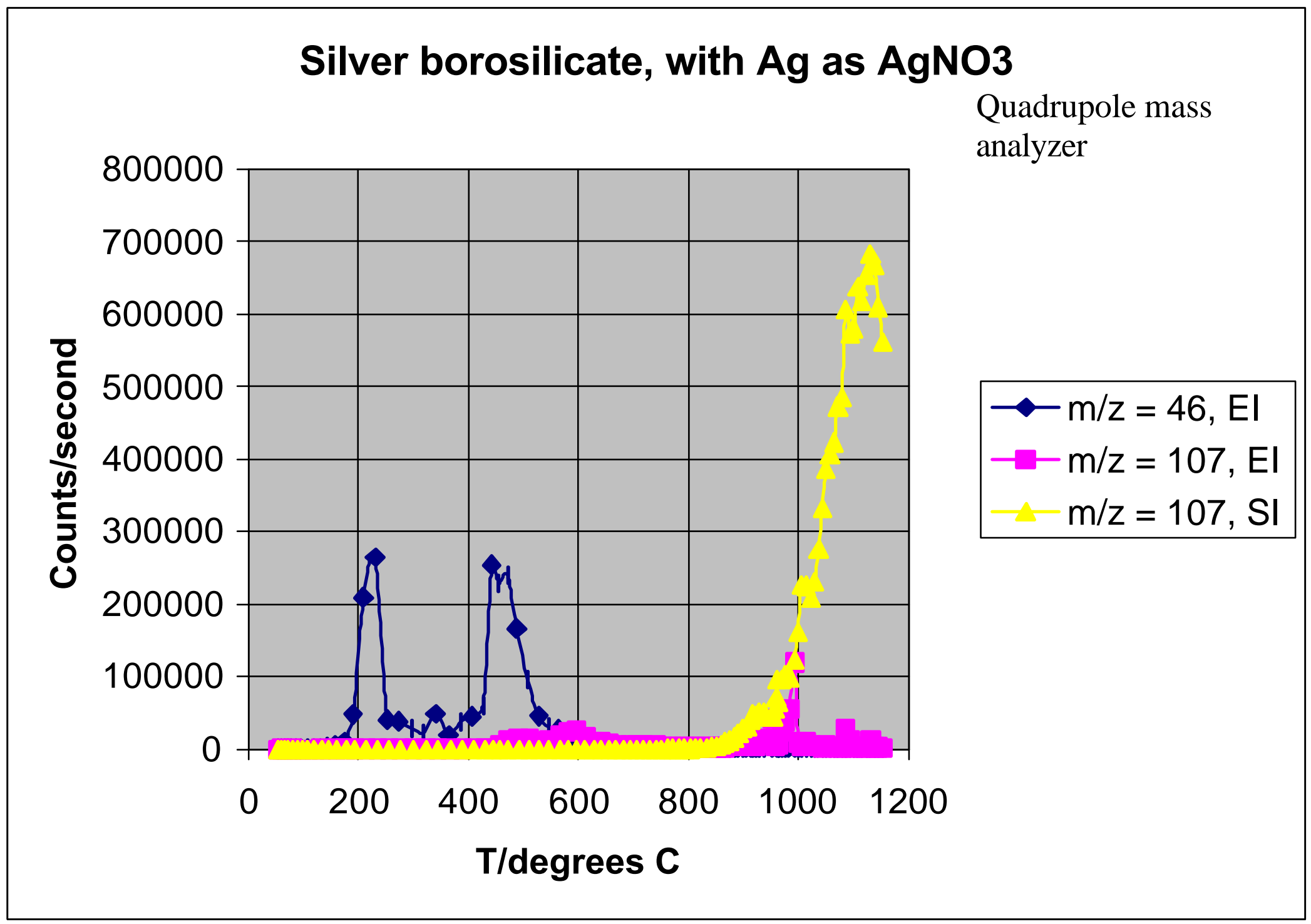

Figure 4. Plot of experimental data from the study of the high temperature chemistry of silver borosilicate with silver added as silver nitrate. 